\title{
Serum uric acid in patients with acute ST-elevation myocardial infarction
}

\author{
Li Chen, Xian-lun Li, Wei Qiao, Zhou Ying, Yan-li Qin, Yong Wang, Yu-jie Zeng, Yuan-nan Ke \\ Department of Cardiology, China-Japan Friendship Hospital, Beijing 100029, China
}

Corresponding Author: Xian-lun Li, Email: lixianlun@hotmail.com

BACKGROUND: Few studies investigated serum uric acid levels in patients with acute STelevation myocardial infarction (STEMI). The study was to assess the clinical value of serum uric acid levels in patients with acute ST-elevation myocardial infarction (STEMI).

METHODS: Totally 502 consecutive patients with STEMI were retrospectively studied from January 2005 to December 2010. The level of serum lipid, echocardiographic data and in-hospital major adverse cardiovascular events (MACE) in patients with hyperuricemia $(n=119)$ were compared with those in patients without hyperuricemia $(n=383)$. The relationship between the level of serum uric acid and the degree of diseased coronary artery was analyzed. All data were analyzed with SPSS version 17.0 software for Student's $t$ test, the Chi-square test and Pearson's correlation coefficient analysis.

RESULTS: Serum uric acid level was positively correlated with serum triglyceride level. Hyperlipidemia was more common in hyperuricemia patients than in non-hyperuricemia patients (43.7\% vs. $33.7 \%, P=0.047)$, and serum triglyceride level was significantly higher in hyperuricemia patients (2.11 \pm 1.24 vs. $1.78 \pm 1.38, P=0.014)$. But no significant association was observed between serum uric acid level and one or more diseased vessels $(P>0.05)$. Left ventricular end-diastolic diameter (LVEDd) was larger in hyperuricemia patients than in non-hyperuricemia patients (53.52 \pm 6.19 vs. $52.18 \pm 4.89, P=0.041)$. The higher rate of left systolic dysfunction and diastolic dysfunction was discovered in hyperuricemia patients (36.4\% vs. $15.1 \%, P<0.001 ; 68.2 \%$ vs. $55.8 \%$, $P=0.023)$. Also, hyperuricemia patients were more likely to have in-hospital MACE $(P<0.05)$.

CONCLUSIONS: Serum uric acid level is positively correlated with serum triglyceride level, but not with the severity of coronary artery disease. Hyperuricemia patients with STEMI tend to have a higher rate of left systolic dysfunction and diastolic dysfunction and more likely to have more in -hospital MACE.

KEY WORDS: Acute ST-elevation myocardial infarction; Serum uric acid; Triglyceride; Coronary angiography; Echocardiography; Left ventricular systolic dysfunction; Left ventricular diastolic dysfunction; Major adverse cardiovascular events

\section{INTRODUCTION}

Clinical and epidemiological studies have proved that serum uric acid (SUA) is significantly correlated with cardiovascular disease. Increased SUA is significantly associated with the occurrence and mortality of coronary artery disease. ${ }^{[1-3]}$ But few studies have investigated serum uric acid levels in patients with acute ST-elevation myocardial infarction (STEMI). This study was undertaken to assess the clinical value of serum uric acid levels in patients with STEMI by comparing their clinical characteristics, coronary angiographic and echocardiographic data and in-hospital outcomes. 


\section{METHODS \\ Patients}

A total of 502 consecutive patients (age 27-89 years) with STEMI were enrolled in this study from January 2005 to December 2010 at the Department of Cardiology, China-Japan Friendship Hospital. All of the patients were divided into two groups according to their levels of SUA. In this series of patients, 119 had hyperuricemia and 383 had non-hyperuricemia. The patients who had liver and kindey diseases, gout, alcoholism and violent exercise were excluded. The diagnosis of patients with acute myocardial infarction (AMI) followed the WHO criteria ${ }^{[4]}$ All patients were given standard treatment for AMI.

\section{Laboratory measurements}

Blood samples were collected next morning for examination on an empty stomach from each patient. SUA, total cholesterol (TC), triglyceride (TG), high density lipoprotein-cholesterol (HDL-C) and low density lipoprotein-cholesterol (LDL-C) were tested with a 7600 automatic biochemistry analyzer (Hitachi Limitied Corporation, Japan). Baseline SUA $\geq 420 \mu \mathrm{mol} / \mathrm{L}$ for men and $\geq 357 \mu \mathrm{mol} / \mathrm{L}$ for women were diagnosed with hyperuricemia. ${ }^{[5]}$

\section{Coronary angiography}

Coronary angiography was examined with Philips V 5000 (Netherlands) and by the Judkin protocol. Coronary stenosis $\geq 50 \%$ was defined as a positive lesion, and one or more of the 3 major coronary arteries was measured. The levels of SUA in diseased vessels were analyzed.

\section{Echocardiography}

Echocardiographic analysis was performed with
Philips IE33 (USA). All patients were examined within one week after admission. Left ventricular end-diastolic diameter (LVEDd) $>55 \mathrm{~mm}$ for men and $>50 \mathrm{~mm}$ for women were regarded as the diagnostic criteria of left ventricular enlargement. Left ventricular ejection fraction (LVEF) $<50 \%$ was considered as left ventricular systolic dysfunction. The early diastolic peak velocities (E) and the late diastolic peak velocities (A) of mitral valve flow were measured, and $\mathrm{E} / \mathrm{A}<1$ was defined as left ventricular diastolic dysfunction.

\section{Statistical analysis}

All data were analyzed by SPSS version 17.0 software. Quantitative data were expressed as mean \pm standard deviation and analyzed by Student's $t$ test. The Chi-square test was used to analyze the nonparametric data. The relationship between SUA and serum lipid was analyzed using Pearson's correlation coefficient analysis. $P<0.05$ was considered statistically significant.

\section{RESULTS \\ Patients' characteristics}

Clinical characteristics of patients are shown in Table 1. There was no significant difference in age and the incidences of hypertension, diabetes mellitus and previous coronary artery disease between the two groups. But there was a significant difference in the incidences of dyslipidemia $(43.7 \%$ vs. $33.7 \%$, $\left.\chi^{2}=3.951, P=0.047\right)$ and the level of TG $(2.11 \pm 1.24 \mathrm{vs}$. $1.78 \pm 1.38, t=2.466, P=0.014)$ between patients with and without hyperuricemia. The levels of TC, HDL-C and LDL-C showed no significant difference between the two groups $(P>0.05)$. There was a significant positive correlation between SUA and TG ( $r=0.11$, $P=0.018$ ), whereas there was a significant negative

Table 1. Comparison of baseline clinical characteristics between the two groups

\begin{tabular}{|c|c|c|c|c|}
\hline Variables & Non-hyperuricemia $(n=383)$ & Hyperuricemia $(n=119)$ & $t / \chi^{2}$ & $P$ \\
\hline Age & $61.19 \pm 14.06$ & $61.51 \pm 14.01$ & 1.220 & 0.83 \\
\hline Sex $($ male $\%)$ & $335(87.5)$ & $82(68.9)^{*}$ & 5.235 & $<0.001$ \\
\hline Hypertension (\%) & $197(51.4)$ & $60(50.4)$ & 0.037 & 0.846 \\
\hline Diabetes mellitus (\%) & $110(28.9)$ & $41(34.5)$ & 1.341 & 0.247 \\
\hline Dyslipidemia (\%) & $129(33.7)$ & $52(43.7)^{*}$ & 3.951 & 0.047 \\
\hline Previous coronary artery disease $(\%)$ & $13(3.4)$ & $9(7.6 \%)$ & 3.765 & 0.052 \\
\hline \multicolumn{5}{|l|}{ Laboratory analysis } \\
\hline $\mathrm{SUA}(\mu \mathrm{mol} / \mathrm{L})$ & $289.50 \pm 73.20$ & $451.87 \pm 100.67^{*}$ & 6.307 & $<0.001$ \\
\hline $\mathrm{TC}(\mathrm{mmol} / \mathrm{L})$ & $4.80 \pm 1.01$ & $5.05 \pm 1.29$ & 1.918 & 0.057 \\
\hline $\mathrm{TG}(\mathrm{mmol} / \mathrm{L})$ & $1.78 \pm 1.38$ & $2.11 \pm 1.24^{*}$ & 2.466 & 0.014 \\
\hline HDL-C (mmol/L) & $1.02 \pm 0.35$ & $1.00 \pm 0.29$ & 0.714 & 0.476 \\
\hline LDL-C (mmol/L) & $2.90 \pm 0.79$ & $3.04 \pm 1.03$ & 1.426 & 0.156 \\
\hline
\end{tabular}


Table 2. Correlation analysis between serum uric acid and serum lipid

\begin{tabular}{lll}
\hline Variables & $r$ & $P$ \\
\hline TC & 0.083 & 0.066 \\
TG & 0.11 & $0.018^{*}$ \\
HDL-C & -0.096 & $0.033^{*}$ \\
LDL-C & 0.031 & 0.486 \\
\hline
\end{tabular}

correlation between SUA and HDL-C ( $r=-0.096$, $P=0.033$ ) (Table 2).

\section{Findings of coronary angiography}

Coronary angiography was performed in 431 patients with STEMI. There was no significant difference in SUA level in patients with different diseased vessels $(P>0.05)$ (Table 3).

\section{Echocardiographic findings}

Echocardiography was performed in 110 patients with hyperuricemia and 317 patients with nonhyperuricemia. Left ventricular end-diastolic diameter was significantly larger in hyperuricemia patients than that in non-hyperuricemia patients $(53.52 \pm 6.19$ vs. $52.18 \pm 4.89$, $t=2.062, P=0.041)$. In addition, there was a significant difference in left ventricular enlargement $(49.1 \%$ vs. $\left.27.4 \%, \chi^{2}=7.300, P<0.001\right)$ and left ventricular ejection fraction between the two groups $(54.44 \pm 12.74$ vs. $58.73 \pm 9.69, t=3.224, P=0.002$ ) (Table 4$)$.

\section{In-hospital prognosis}

The average days in hospital stay were similar in both groups (average hospital stay was $12.06 \pm 5.70$ days). Hyperuricemia patients had more in-hospital MACE including heart failure, cardiogenic shock, stroke, acute renal failure and mortality compared with nonhyperuricemia patients $(P<0.05)$. In addition, there were no differences in rates of arrhythmia and atrioventricular block between the two groups $(P>0.05)$ (Table 5).

\section{DISCUSSION}

In this study, SUA levels were correlated positively with serum TG levels $(r=0.11, P=0.018)$, but correlated negatively with HDL-C levels. Serum TG levels were significantly higher in patients with hyperuricemia compared with controls $(2.11 \pm 1.24$ vs. $1.78 \pm 1.38, P$ $=0.014)$. The levels of HDL-C were lower in nonhyperuricemia patients, but there was no significant difference $(1.00 \pm 0.29$ vs. $1.02 \pm 0.35, P=0.476)$. Epidemiologic studies have shown that SUA level is affected by many factors, and it is closely related to the metabolism of lipids. Hyperuricemia is often associated with dyslipidemia, especially hypertriglyceridemia. ${ }^{[6,7]}$ The mechanism of the close relationship between SUA level and lipid metabolism has not been understood completely. It may involve both metabolic and genetic

Table 3. Comparison of serum uric acid level in groups of different diseased vessels

\begin{tabular}{llll}
\hline Variables & One diseased vessel $(n=119)$ & Two diseased vessels $(n=116)$ & Three diseased vessels $(n=196)$ \\
\hline SUA $(\mu \mathrm{mol} / \mathrm{L})$ & $321.94 \pm 91.02$ & $329.72 \pm 106.71$ & $330.67 \pm 106.47$ \\
\hline
\end{tabular}

Table 4. Comparison of echocardiographic features between the two groups

\begin{tabular}{|c|c|c|c|c|}
\hline Echocardiography & Non-hyperuricemia $(n=317)$ & Hyperuricemia $(n=110)$ & $t / \chi^{2}$ & $P$ \\
\hline LVEDd (mm) & $52.18 \pm 4.89$ & $53.52 \pm 6.19^{*}$ & 2.062 & 0.041 \\
\hline LVEDd enlarge $(\%)$ & $87(27.4)$ & $54(49.1)^{*}$ & 7.300 & $<0.001$ \\
\hline $\operatorname{LVEF}(\%)$ & $58.73 \pm 9.69$ & $54.44 \pm 12.74^{*}$ & 3.224 & 0.002 \\
\hline
\end{tabular}

Table 5. Comparison of clinical outcomes between in-hospital patients

\begin{tabular}{|c|c|c|c|c|}
\hline Variables & Non-hyperuricemia $(n=383)$ & Hyperuricemia $(n=119)$ & $t / \chi^{2}$ & $P$ \\
\hline Length of hospital stay (d) & $11.85 \pm 5.17$ & $12.91 \pm 7.04$ & 1.515 & 0.132 \\
\hline Arrhythmia (\%) & $25(6.5)$ & $13(10.9)$ & 2.509 & 0.113 \\
\hline Atrioventricular block (\%) & $20(5.2)$ & $8(6.7)$ & 0.388 & 0.533 \\
\hline Cardiogenic shock $(\%)$ & $21(5.5)$ & $14(11.8)^{*}$ & 5.524 & 0.019 \\
\hline Heart failure $(\%)$ & $62(16.2)$ & $57(47.9)^{*}$ & 6.481 & $<0.001$ \\
\hline $\mathrm{LVEF}<50 \%(\%)$ & $48(15.1)$ & $40(36.4)^{*}$ & 5.845 & $<0.001$ \\
\hline $\mathrm{E} / \mathrm{A}<1(\%)$ & $177(55.8)$ & $75(68.2)^{*}$ & 5.827 & 0.023 \\
\hline Stroke $(\%)$ & $0(0)$ & $5(4.2)^{*}$ & 6.254 & $<0.001$ \\
\hline Acute renal failure $(\%)$ & $0(0)$ & $6(5.0)^{*}$ & 6.545 & $<0.001$ \\
\hline Overall mortality (\%) & $23(6.0)$ & $15(12.6)^{*}$ & 3.129 & 0.017 \\
\hline
\end{tabular}


defects, which mean lipid metabolic disorders along with purine metabolic disorders. Meanwhile afferent and efferent arterioles of glomerulus can be involved when lipid metabolic disorders occur, resulting in the stenosis or occlusion of arteries and transrenal excretion of uric acid.

Uric acid promotes the development of atherosclerosis, while causing a variety of cardiovascular events. The possible mechanisms vary ${ }^{[8]}$ The high level of SUA promotes the oxidation of LDL-C and the peroxidation of lipid. It also increases the formation of oxygen radicals in inflammatory reaction. In addition, the high level of SUA also increases platelet aggregation and the formation of uric acid crystals. The deposition of uric acid in the arterial wall could damage the tunica intima of arteries, promoting coronary thrombosis. Studies proved that SUA levels were significantly higher in patients with coronary artery disease than those in patients without this disease, but SUA level and the degree of diseased coronary arteries were not significantly correlated. ${ }^{[9,10]}$ The results of the present study showed that SUA level in patients with STEMI increased with the rise of the number of diseased coronary arteries, but there was no significant difference between the groups $(P>0.05)$. Hence SUA level was not significantly related to the stenosis of coronary arteries.

In the present study, LVEDd was significantly larger in hyperuricemia patients with STEMI than in the control group (53.52 \pm 6.19 vs. $52.18 \pm 4.89, P=0.041$ ). Left ventricular ejection fraction was significantly lower than that in the non-hyperuricemia group $(54.44 \pm 12.74$ vs. $58.73 \pm 9.69, P=0.002$ ), and the proportions of LVEF $<50 \%$ and $\mathrm{E} / \mathrm{A}<1$ were significantly higher in the hyperuricemia patients $(36.4 \%$ vs. $15.1 \%, P<0.001$; $68.2 \%$ vs. $55.8 \%, P=0.023)$. Simultaneously, the incidence of MACE including heart failure, cardiogenic shock and death was significantly higher than that in the control group $(P<0.05)$ during hospitalization (average hospital stay was $12.06 \pm 5.70 \mathrm{~d}$ ). After AMI, patients with hyperuricemia were found to be more susceptible to left ventricular remodeling as well as left ventricular systolic or diastolic dysfunction. Thus the incidence of MACE was increased. Hyperuricemia is also thought to increase oxidative stress and activate inflammatory cytokines, while inducing cardiomyocyte apoptosis, then promoting myocardial remodeling. Furthermore, AMI may cause tissue hypoxia and hypoperfusion, which could enhance the roles of xanthine oxidase and oxidative stress; subsequently SUA level increases in turn. Finally a vicious cycle in which cardiac function gets worse is formed. ${ }^{[11]}$ Hyperuricemia is recognized a good predictor of heart failure and death in patients after AMI. ${ }^{[12-14]}$ Compared with such markers as brain natriuretic peptide and high-sensitivity $\mathrm{C}$-reactive protein, which have been widely used in assessing the prognosis of AMI, ${ }^{[15,16]}$ whether SUA could be an independent predictor of heart failure and death after myocardial infarction remains to be confirmed by further studies.

In summary, SUA level is positively correlated with serum TG level, but it is not significantly associated with the stenosis degree of the coronary artery. Hyperuricemia patients with acute myocardial infarction have a higher rate of left ventricular systolic, diastolic dysfunction and MACE (including heart failure, death). Reduction of the concentration of uric acid may decrease the incidence of heart failure and death in patients with myocardial infarction.

\section{Funding: None.}

Ethical approval: Not needed.

Conflicts of interest: The authors have no financial or other conflicts of interest regarding this article.

Contributors: Chen L drafted the manuscript. All authors read and approved the final manuscript.

\section{REFERENCES}

1 Baker JF, Krishnan E, Chen L, Schumacher HR. Serum uric acid and cardiovascular disease: recent developments, and where do they leave us? Am J Med 2005; 118: 816-826.

2 Brodov Y, Chouraqui P, Goldenberg I, Boyko V, Mandelzweig L, Behar S. Serum uric acid for risk stratification of patients with coronary artery disease. Cardiology 2009; 114: 300-305.

3 Bae MH, Lee JH, Lee SH, Park SH, Yang DH, Park HS, et al. Serum uric acid as an independent and incremental prognostic marker in addition to N-terminal pro-B-type natriuretic peptide in patients with acute myocardial infarction. Circ J 2011; 75: 1440-1477.

4 Thygesen K, Alpert JS, White HD; Joint ESC/ACCF/AHA/ WHF Task Force for the Redefinition of Myocardial Infarction, Jaffe AS, Apple FS, et al. Universal definition of myocardial infarction. Circulation 2007; 116: 2634-2653.

5 Kanbay M, Solak Y, Dogan E, Lanaspa MA, Covic A. Uric acid in hypertension and renal disease: the chicken or the egg? Blood Purif 2010; 30: 288-295. Epub 2010 Nov 20.

6 Rathmann W, Haastert B, Icks A, Giani G, Roseman JM. Tenyear change in serum uric acid and its relation to changes in other metabolic risk factors in young black and white adults: the CARDIA study. Eur J Epidemiol 2007; 22: 439-445.

7 Lippi G, Montagnana M, Luca Salvagno G, Targher G, Cesare Guidi G. Epidemiological association between uric acid concentration in plasma, lipoprotein (a) and the traditional lipid 
profile. Clin Cardiol 2010; 33: 76-80.

8 Ruggiero C, Cherubini A, Ble A, Bos AJ, Maggio M, Dixit VD, et al. Uric acid and inflammatory markers. Eur Heart J 2006; 27: 1174-1181.

9 Petersen TS, Madsen TV, Jespersen JB, Larsen A, Schmidt EB, Christensen JH. Uric acid in patients with angiographically documented coronary heart disease. Acta Cardiol 2006; 6: 525529.

10 Gur M, Yilmaz R, Demirbag R, Aksoy N. Relation of serum uric acid levels with the presence and severity of angiographic coronary artery disease. Angiology 2008; 59: 166-171.

11 Hare JM, Johnson RJ. Uric acid predicts clinical outcomes in heart failure: insights regarding the role of xanthine oxidase and uric acid in disease pathophysiology. Circulation 2003; 107: 1951-1953.

12 Kojima S, Sakamoto T, Ishihara M, Kimura K, Miyazaki S, Yamagishi M, et al. Prognostic usefulness of serum uric acid after acute myocardial infarction(the Japanese Acute Coronary Syndrome Study). Am J Cardiol 2005; 96: 489-495.
13 Car S, Trkulja V. Higher serum uric acid on admission is associated with higher short-term mortality and poorer longterm survival after myocardial infarction: retrospective prognostic study. Croat Med J 2009; 50: 559-566.

14 Nadkar MY, Jain VI. Serum uric acid in acute myocardial infarction. J Assoc Physicians India 2008; 56: 759-762.

15 Windhausen F, Hirsch A, Sanders GT, Cornel JH, Fischer J, van Straalen JP, et al. N-terminal pro-brain natriuretic peptide for additional risk stratification in patients with non-ST-elevation acute coronary syndrome and an elevated troponin $\mathrm{T}$ : an Invasive versus Conservative Treatment in Unstable coronary Syndromes (ICTUS) substudy. Am Heart J 2007; 153: 485-492.

16 Matsui S, Ishii J, Kitagawa F, Kuno A, Hattori K, Ishikawa $\mathrm{M}$, et al. Pentraxin 3 in unstable angina and non-ST-segment elevation myocardial infarction. Atherosclerosis 2010; 210: 220-225. Epub 2009 Nov 5.

Received October 9, 2011 Accepted after revision February 1, 2012 